\title{
-NOTES-
}

\section{SOME MAXIMUM PRINCIPLES FOR NONLINEAR ELLIPTIC BOUNDARY-VALUE PROBLEMS*}

\author{
By PHILIP W. SCHAEFER (University of Tennessee, Knoxville)
}

\begin{abstract}
The Hopf maximum principles are utilized to obtain maximum principles for functions which are defined on solutions of nonlinear, second-order elliptic equations subject to Dirichlet, Robin, or mixed boundary conditions. The principles derived may be used to deduce bounds on important quantities in physical problems of interest.
\end{abstract}

1. Introduction. Several authors have recently developed maximum principles for certain functions defined on solutions of linear and nonlinear elliptic boundary-value problems in order to obtain differential inequalities which lead to bounds on quantities important in various physical problems. One should consult $[2-6,9,10]$ in the references for this development. Until [6], these results were accomplished for boundary-value problems (primarily Dirichlet problems) for equations of the form

$$
\Delta u+\lambda \rho(x) f(u)=0,
$$

where $\Delta$ is the Laplace operator, or special cases thereof (usually $\lambda=1, \rho(x) \equiv$ 1). In [6] the authors considered the problem of torsional creep, i.e.

$$
\left(g\left(q^{2}\right) u_{, i}\right)_{, i}+2=0 \quad \text { in } D, \quad u=0 \quad \text { on } \partial D
$$

where $q=|\operatorname{grad} u|$, and developed isoperimetric bounds for the maximum stress and the torsional stiffness.

In this paper we develop maximum principles for a variety of boundary-value problems for the nonlinear equation

$$
\left(g(u) u_{, i}\right)_{, i}+f(u)=0 .
$$

These results are presented in Theorems 1,2 and 3 of Sec. 2. One can, in fact, follow the procedure in [10] and obtain principles for the inhomogeneous equation

$$
\left(g(u) u_{, i}\right)_{, i}+\rho(x) f(u)=0 .
$$

Because of the computational complexity, we indicate only the fundamental changes and then state the results as Theorems 4 and 5. Remarks concerning extensions and improvements are given in Sec. 3. We refer the reader to [1] and references cited therein for problems which give rise to equations of the form

\footnotetext{
* Received August 12, 1976; revised version received November 3, 1976.
} 
(1.2). One is referred to [5] for various techniques one might apply to the resulting differential inequalities to obtain bounds on the solution or the gradient of the solution and to [8] and the references cited there for other principles and bounding techniques.

We shall be primarily interested in the following problems. Let $D$ be a convex domain in the plane bounded by a sufficiently smooth curve $\partial D$. We assume that $u$ is a solution of

$$
\begin{gathered}
\left(g(u) u_{, i}\right)_{, i}+f(u)=0 \quad \text { in } D \\
u=0 \text { on } \Gamma_{1}, \quad \Gamma_{1} \neq \emptyset \\
\partial u / \partial n=0 \quad \text { on } \Gamma_{2}, \quad \Gamma_{1} \cup \Gamma_{2}=\partial D
\end{gathered}
$$

or

$$
\begin{gathered}
\left(g(u) u_{, i}\right)_{, i}+f(u)=0 \quad \text { in } D \\
\partial u / \partial n+\alpha u=0 \quad \text { on } \partial D, \quad \alpha>0
\end{gathered}
$$

where the comma notation denotes partial differentiation, the repeated index denotes summation, and $\partial u / \partial n$ denotes the outward normal derivative. In both problems, we assume that $f$ is a $C^{1}$ function and $g$ is a positive $C^{2}$ function of $u$. We note that in (MP), $\Gamma_{2}$ may be empty. If $\Gamma_{2}=\emptyset$ in (MP), then we refer to the resulting problem as (DP), the Dirichlet problem. For (DP), the result in Theorem 2 is valid in higher dimensions as well.

2. Maximum principles. Let $u$ be a sufficiently smooth solution of (1.1). We define

$$
\Phi=\left(g(u) u_{, i}\right)\left(g(u) u_{, i}\right)+2 \int_{0}^{u} f(\eta) g(\eta) d \eta
$$

and compute

$$
\begin{gathered}
\Phi_{, k}=2\left(g u_{, i}\right)\left(g u_{, i}\right)_{, k}+2 f\left(g u_{, k}\right) \\
\Phi_{, k k}=2\left(g u_{, i}\right)_{, k}\left(g u_{, i}\right)_{, k}+2\left(g u_{, i}\right)\left(g u_{, i}\right)_{, k k}+2 f^{\prime} g u_{, k} u_{, k}+2 f\left(g u_{, k}\right)_{, k}
\end{gathered}
$$

where the prime denotes differentiation with respect to the argument and we have suppressed the argument. From (2 2) and Schwarz's inequality, it follows that

$$
2\left(g u_{, i}\right)_{, k}\left(g u_{, i}\right)_{, k} \geq \frac{1}{2\left(g u_{, j}\right)\left(g u_{, j}\right)}\left[\Phi_{, k} \Phi_{, k}-4 f g u_{, k} \Phi_{, k}+4 f^{2} g^{2} u_{, k} u_{, k}\right] .
$$

Consequently, by (1.1) and (2.4) we can write

$$
\Phi_{, k k}+\frac{L_{k} \Phi_{, k}}{|\nabla u|^{2}} \geq 2\left(g u_{, i}\right)\left(g u_{, i}\right)_{, k k}+2 f^{\prime} g|\nabla u|^{2},
$$

where

$$
L_{k}=\frac{1}{2 g^{2}}\left[4 f g u_{, k}-\Phi_{, k}\right] .
$$

Now consider the identity

$$
\left(g u_{, i}\right)\left(g u_{, i}\right)_{, k k}=g^{2} u_{, k k i} u_{, i}+2 g g^{\prime} u_{, i} u_{, k} u_{, i k}+g g^{\prime \prime} u_{, i} u_{, i} u_{, k} u_{, k}+g g^{\prime} u_{, i} u_{, i} u_{, k k} .
$$


From (1.1) it follows that

$$
\begin{gathered}
d u_{, k k}=-f-g^{\prime} g u_{, k} u_{, k} \\
g^{2} u_{, k k i}=\left(f g^{\prime}-f^{\prime} g\right) u_{, i}+\left(g^{\prime 2}-g g^{\prime \prime}\right) u_{, i} u_{, k} u_{, k}-2 g g^{\prime} u_{, k} u_{, k i}
\end{gathered}
$$

and hence, on substituting into (2.6), that

$$
2\left(g u_{, i}\right)\left(g u_{, i}\right)_{, k k}=-2 f^{\prime} g|\nabla u|^{2} .
$$

Thus we have

$$
\Phi_{, k k}+\frac{L_{k} \Phi_{, k}}{|\nabla u|^{2}} \geq 0
$$

and by the maximum principle for elliptic operators [7], we arrive at our first result.

TheOrem 1. If $u$ is a $C^{3}$ solution of (1.1) in $D$, where $f$ is a $C^{1}$ and $g$ is a positive $C^{2}$ function of $u$, then

$$
\Phi=[g(u)]^{2}|\nabla u|^{2}+2 \int_{0}^{u} f(\eta) g(\eta) d \eta
$$

takes its maximum either on $\partial D$ or at a critical point of $u$.

We note that Theorem 1 is valid for $n>2$ as there was no dependence on dimension. In fact, when $n=2$, we may use (1.1) and (2.2) to obtain an identity for the terms $2\left(g u_{, i}\right)_{, k}\left(g u_{, i}\right)_{, k}$ so that $\Phi$ satisfies an elliptic equation rather than inequality (2.9). In this regard, see [9].

Let us now consider (MP). We shall show that $\Phi$ cannot attain its maximum on $\partial D$ unless it is attained at a critical point of $u$ which is on $\mathrm{I}_{2}$.

Suppose that $\Phi$ takes its maximum at $P \in \Gamma_{1}$. Then $P$ cannot be a critical point of $u$. Since $u=0$ on $\Gamma_{1}$, we have $|\nabla u|=|\partial u / \partial n|$ and

$$
\partial \Phi / \partial n=2 g^{2} u_{n} u_{n n}+2 g g^{\prime} u_{n}{ }^{3}+2 f g u_{n},
$$

where $u_{n}$ denotes the outward normal derivative. Introducing normal coordinates in the neighborhood of the boundary, we can write

$$
\Delta u \equiv u_{n n}+k u_{n}=-\frac{f}{g}-\frac{g^{\prime}}{g}|\nabla u|^{2}
$$

where $k$ denotes the curvature of the boundary. Thus it follows that

$$
\partial \Phi / \partial n=-2 k g^{2} u_{n}^{2},
$$

and since $D$ is convex, that $\partial \Phi / \partial n \leq 0$ at $P$. This, however, contradicts Hopf's second maximum principle [7].

We now suppose that $\Phi$ takes its maximum at $P \in \Gamma_{2}$ and that $P$ is not a critical point of $u$. Since $\partial u / \partial n=0$ on $\Gamma_{2}$, we have $|\nabla u|=|\partial u / \partial s|$ and

$$
\partial \Phi / \partial n=2 g^{2} u_{s} u_{s n},
$$

where $u_{s}$ denotes the tangential derivative of $u$. In terms of normal coordinates in the neighborhood of the boundary, we have

so that on $\mathrm{\Gamma}_{2}$

$$
u_{s n}=u_{n s}-k u_{s},
$$

$$
\partial \Phi / \partial n=-2 k g^{2} u_{s}^{2}
$$


Thus, we again arrive at a contradiction to the second maximum principle when $D$ is convex. We state our conclusion as

THEOREM 2. If $u$ is a $C^{3}$ solution of either (DP) or (MP), then $\Phi$ given by (2.1) takes its maximum at a critical point of $u$.

We observe that the conclusion of Theorem 2 is also valid in higher dimensions when $u$ is subject to a Dirichlet condition on $\partial D$. For $n>2$, we have, instead of (2.11),

$$
\Delta u \equiv u_{n n}+(n-1) K u_{n}=-\frac{f}{g}-\frac{g^{\prime}}{g}|\nabla u|^{2},
$$

where $K$ denotes the average curvature of $\partial D$. Substitution into $(2.10)$ now results in

$$
\partial \Phi / \partial n=-2(n-1) K g^{2} u_{n}{ }^{2} .
$$

Thus if $D$ is a convex region in $n>2$ dimensions, we again conclude that the maximum of $\Phi$ occurs at a critical point of $u$.

We now consider (RP). We shall find that under certain additional assumptions on $f$ and $g, \Phi$ cannot attain its maximum on $\partial D$.

Here we assume that $u$ satisfies the boundary condition

$$
\partial u / \partial n+\alpha u=0, \quad \alpha>0 .
$$

Hence we write

$$
\Phi=g^{2}\left(u_{n}^{2}+u_{s}^{2}\right)+2 \int_{0}^{u} f(\eta) g(\eta) d \eta
$$

and compute

$$
\partial \Phi / \partial n=2 g^{2}\left(u_{n} u_{n n}+u_{s} u_{s n}\right)+2 g g^{\prime}|\nabla u|^{2} u_{n}+2 f g u_{n} .
$$

Introducing normal coordinates, we can write

$$
\Delta u \equiv u_{n n}+k u_{n}+u_{s s}=-\frac{f}{g}-\frac{g^{\prime}}{g}|\nabla u|^{2},
$$

which, together with (2.13) and (2.14), results in

$$
\partial \Phi / \partial n=-2 g^{2}\left\{k \alpha^{2} u^{2}-\alpha u u_{s s}+(\alpha+k) u_{s}^{2}\right\} .
$$

Now suppose that $\Phi$ takes its maximum at $P$ on $\partial D$. Then at $P$

$$
\partial \Phi / \partial s=0,
$$

where

$$
\begin{aligned}
\partial \Phi / \partial s & =2 g^{2}\left(u_{n} u_{n s}+u_{s} u_{s s}\right)+2 g g^{\prime}|\nabla u|^{2} u_{s}+2 f g u_{s} \\
& =2 g u_{s}\left\{g \alpha^{2} u+g u_{s s}+g^{\prime}|\nabla u|^{2}+f\right\}
\end{aligned}
$$

Since $g>0$, either $u_{s}=0$ or the expression in the braces vanishes at $P$.

Case 1: Suppose $u_{s} \neq 0$ at $P$. In this case

$$
g u_{s s}=-\left\{g \alpha^{2} u+g^{\prime}|\nabla u|^{2}+f\right\} .
$$

Now if we ask that $f, g>0$ and $g^{\prime}>0$, then it follows from (2.7) and (2.14) that $u \geq 0$ in $D$ $\cup \partial D$. Hence from (2.17) we have $u_{s s} \leq 0$ and from $(2.15)$ that $\partial \Phi / \partial n \leq 0$ at $P$.

Case 2: Suppose $u_{s}=0$ at $P$. Under the assumption that $\Phi$ takes its maximum at $P$ on $\partial D$, we know that $\Phi_{s s} \leq 0$, where 


$$
\Phi_{s s} \equiv \partial^{2} \Phi / \partial s^{2}=2 g u_{s s}\left\{g \alpha^{2} u+g u_{s s}+g^{\prime}|\nabla u|^{2}+f\right\}
$$

Hence, either

(i) $u_{s s} \geq 0$ and $g \alpha^{2} u+g u_{s s}+g^{\prime}|\nabla u|^{2}+f \leq 0$

or

(ii) $u_{s s} \leq 0$ and $g \alpha^{2} u+g u_{s s}+g^{\prime}|\nabla u|^{2}+f \geq 0$.

As in case 1 , if $f, g>0$ and $g^{\prime}>0$, then $u \geq 0$ in $D \cup \partial D$. Under these conditions (i) is impossible since if $u_{s s} \geq 0$, then

$$
g \alpha^{2} u+g u_{s s}+g^{\prime}|\nabla u|^{2}+f \geq 0 .
$$

Thus we conclude that (ii) holds, i.e., $u_{s s} \leq 0$. From (2.15) we again deduce that $\partial \Phi / \partial n \leq$ 0 at $P$. Therefore, by the second maximum principle [7], we conclude that $\Phi$ cannot take its maximum at $P$ on $\partial D$ and state

THEOREM 3. If $u$ is a $C^{3}$ solution of (RP), where $f$ is a positive $C^{1}$ and $g$ is a positive $C^{2}$ function for which $g^{\prime}>0$, then $\Phi$ given by (2.1) takes its maximum at a critical point of $u$.

Let us now consider the inhomogeneous equation (1.2) where $\rho(x)>0$ in $D$. Following [10] and the derivation in Theorem 1, it is not difficult to show that

$$
\psi=\frac{1}{\rho}\left(g u_{, i}\right)\left(g u_{, i}\right)+2 \int_{0}^{u} f(\eta) g(\eta) d \eta
$$

satisfies

$$
\psi_{, k k}+\frac{H_{k} \psi_{, k}}{\left(g u_{, j}\right)\left(g u_{, j}\right)} \geq\left\{\frac{|\nabla \rho|^{2}}{2 \rho^{3}}-\frac{\Delta \rho}{\rho^{2}}\right\}\left(g u_{, j}\right)\left(g u_{, j}\right),
$$

where

$$
H_{k}=\rho\left\{2 f g u_{, k}-\frac{1}{2} \psi_{, k}\right\}+\frac{\rho_{, k}}{\rho}\left(g u_{, j}\right)\left(g u_{, j}\right) .
$$

Thus we have the following extension of Theorem 1 .

TheOrem 4. If $u$ is a $C^{3}$ solution of (1.2) in $D$, where $f$ is a $C^{1}$ and $g$ is a positive $C^{2}$ function of $u$ and $\rho$ is a positive $C^{2}$ function for which $\Delta \rho \leq 0$ in $D$, then $\psi$ given by (2.18) takes its maximum either on $\partial D$ or at a critical point of $u$.

We note that Theorem 4 is also valid for $n>2$. Furthermore, one can develop the analogue of Theorem 2 for solutions of (1.2) provided additional constraints are imposed on $g, \rho$, and/or the curvature of $\partial D$.

Consider the inhomogeneous mixed problem

$$
\begin{gathered}
\left(g(u) u_{, i}\right)_{, i}+\rho(x) f(u)=0 \quad \text { in } D \\
u=0 \quad \text { on } \Gamma_{1}, \quad \Gamma_{1}=\emptyset \\
\partial u / \partial n=0 \quad \text { on } \Gamma_{2}, \quad \Gamma_{1} \cup \Gamma_{2}=\partial D
\end{gathered}
$$

where $g, f$, and $\rho$ are as stated in Theorem 4 . Then $\psi$ takes its maximum on $\partial D$ or at a critical point of $u$. But, following the derivation in Theorem 2, we find that on $\partial D$

$$
\partial \psi / \partial n=-\frac{g^{2}|\nabla u|^{2}}{\rho}\left\{2 k+\frac{\partial}{\partial n}(\ln \rho)\right\}
$$

whether we assume that $\psi$ takes its maximum at $P$ on $\Gamma_{1}$ or $\Gamma_{2}$. Thus, if $\partial \psi / \partial n \leq 0$ at $P$ on $\partial D$, we can deduce that $\psi$ takes its maximum at a critical point. This requirement will be 
satisfied if the geodesic curvature $k_{g}$ of $\partial D$ (see [10]), where

$$
k_{g}=\rho^{-1 / 2}\left\{2 k+\frac{\partial}{\partial n}(\ln \rho)\right\},
$$

is nonnegative or if $g$ vanishes for points on the boundary, as when $g$ is a power function and $u$ is subject to Dirichlet conditions only. We state this extension formally as

THEOREM 5. If $u$ is a $C^{3}$ solution of (IMP) and if $[g(u)]^{2} k_{g} \geq 0$ on $\partial D$, where $k_{g}$ is the geodesic curvature of $\partial D$, then $\psi$ given by (2.18) takes its maximum at a critical point of $u$.

An extension of Theorem 3 to the inhomogeneous equation (1.2)-even in the case $g(u) \equiv 1$ - has not been accomplished.

3. Concluding remarks. Obviously, the principles and applications in $[2-5,9,10]$ are "covered" when $g(u) \equiv 1$ here. Moreover, one could follow [9] and seek other functions of the solution $u$ that satisfy maximum principles of the type presented here. This appears to involve an undesirable amount of complexity.

We also note that a wide range of mixed boundary value problems could be considered. For example: if $u$ is a $C^{3}$ solution of $(1.1)$ in $D$ and satisfies a Dirichlet condition on a portion of the boundary and a Robin condition on the remainder of the boundary, then $\Phi$ given by (2.1) attains its maximum at a critical point of $u$ provided that $f$ and $g$ satisfy the conditions cited in Theorem 3.

Let us now consider a simple illustration in which we determine a bound for the gradient of the solution of a nonlinear Robin problem at any point in the domain in terms of the maximum value of the solution function. Consider the problem

$$
\begin{aligned}
\Delta u+u_{i,} u_{, i}+1=0 & \text { in } D \\
\partial u / \partial n+\alpha u=0 & \text { on } \partial D
\end{aligned}
$$

which can be rewritten as

$$
\begin{aligned}
& \left(e^{u} u_{, i}\right)_{, i}+e^{u}=0 \text { in } D \\
& \partial u / \partial n+\alpha u=0 \text { on } \partial D \text {. }
\end{aligned}
$$

With $f(u)=e^{u}=g(u)$, it follows from Theorem 3 that

$$
e^{2 u}|\nabla u|^{2}+2 \int_{0}^{u} e^{2 t} d t \leq\left. 2 \int_{0}^{u} e^{2 t} d t\right|_{\max }
$$

or

$$
|\nabla u|^{2} \leq e^{2 u_{m}}-1
$$

where $u_{m}$ is the maximum value of $u$ in $D \cup \partial D$.

\section{REFERENCES}

[1] V. Komkov, Certain estimates for solutions of nonlinear elliptic differential equations applicable to the theory of thin plates, SIAM J. Appl. Math 28, 24-34 (1975)

[2] L. E. Payne. Bounds for the maximum stress in the Saint Venant torsion problem, Indian J. Mech. and Math. Special Issue, 51-59(1968)

[3] L. F. Payne and I. Stakgold. On the mean value of the fundamental mode in the fixed membrane problem, Appl. Anal. 3, 295-306 (1973) 
[4] L. E. Payne and I. Stakgold, Nonlinear problems in nuclear reactor analysis, in Proc. Conference on Nonlinear Problems in Physical Sciences and Biology, Springer Lecture Notes in Math. No. 322, 298-307 (1972)

[5] L. E. Payne, R. P. Sperb, and I. Stakgold, On Hopf type maximum principles for convex domains (to appear)

[6] L. E. Payne and G. A. Philippin, Some applications of the maximum principle in the problem of torsional creep (to appear)

[7] M. H. Protter and H. F. Weinberger, Maximum principles in differential equations, Prentice-Hall, Inc. (1967)

[8] M. H. Protter and H. F. Weinberger, A maximum principle and gradient bounds for linear elliptic equations, Indiana U. Math. J. 23, 239-249 (1973)

[9] P. W. Schaefer and R. P. Sperb, Maximum principles for some functionals associated with the solution of elliptic boundary value problems, Arch. Rational Mech. Anal. 61, 65-76 (1976)

[10] P. W. Schaefer and R. P. Sperb, Maximum principles and bounds in some inhomogeneous elliptic boundary value problems, SIAM J. Math. Anal. 8 (1977) 